Clinical Reviews in Allergy \& Immunology

(C) Copyright 2003 by Humana Press Inc. 1080-0549/03/249-258/\$25.00

\title{
Severe Bronchiolitis in Children
}

\author{
Sanjay Jhawar \\ Pediatrics Pulmonary Division, University of California Davis Medical Center, Sacramento, CA; \\ E-mail: sjhawar@ucdavis.edu
}

Bronchiolitis is a common, acute, contagious lower respiratory tract illness of infants and young children. The majority of cases are secondary to respiratory syncytial virus. There are a number of risk factors for severe disease, including children less than six weeks of age and patients with atopy and/or asthma. The management requires vigilant monitoring and highquality supportive care, including impressive use of fluids and nutritional support. Further research on anti-virals is essential to prevent respiratory syncytial virus induced bronchiolitis. Fortunately, the prognosis for the majority of normal infants who develop bronchiolitis is good.

\section{Index Entries:}

Respiratory syncytial virus; bronchiolitis; cystic fibrosis; dehydration; coryza.

\section{Introduction}

Bronchiolitis is a common, acute, contagious lower respiratory tract illness of infants and young children. Severe bronchiolitis with involvement of small airways and the gas exchanging units in the lung (terminal and respiratory bronchioles) is associated with significant respiratory distress, low oxygen saturation, and respiratory failure, which may require intubation, assisted ventilation, and intensive care in the hospital. Bronchiolitis may be severe or atypical in mixed viral or viral-bacterial infections, particularly in high-risk infants.

\section{Epidemiology}

Bacteria, e.g., Hemophilus influenzae, are not proven to be the primary cause of bronchiolitis. Respiratory syncytial virus (RSV) causes $80-90 \%$ of cases of bronchiolitis. RSV is the major cause in community outbreaks, whereas other infectious agents account for sporadic disease. Parainfluenza viruses are the second most common isolated agents (1-7). Adenovirus can cause severe bronchiolitis with longlasting pulmonary dysfunction (8).

Bronchiolitis occurs throughout the world, with annual outbreaks. Peak incidence is dur- 
Table 1

Risk Factors for Severe Bronchiolitis

Age $<6$ wk
Prematurity
Bronchopulmonary dysplasia
Hemodynamically significant cardiopulmonary
disease
Congenital lung and airway malformations
Cystic fibrosis
Ciliary dyskinesia syndrome
Immune suppression
History of asthma
Family history of atopic disorders
Environmental factors:
Exposure to environmental tobacco smoke (ETS)
Lower socioeconomic status
Lack of breast feeding
Day-care attendance
Exposure to wood burning stove smoke

ing winter and spring months in temperate and rainy season in tropical climates.

The most affected age group is 2 mo to 2 yr. Eighty percent of all cases occur in the first year of life with a peak between 2 and 6 mo. It is more common and is seen at younger ages in urban areas. Prematurity is a significant risk factor for severe illness. Bronchiolitis is slightly more common in boys, who also experience more severe disease. Low-income families, overcrowding, and lack of breast-feeding are also correlated with more severe disease.

Exposure to environmental tobacco smoke (ETS), particularly maternal smoking, has been shown to be an important risk factor in young infants. Children living in homes where a wood-burning stove is used get bronchiolitis more often.

Although illness is mild in the majority of cases, $10 \%$ of infected infants develop bronchiolitis and up to $5 \%$ require hospital admission. RSV bronchiolitis is highly contagious; during epidemics up to $50 \%$ of inpatients can be affected and the chances increase with increasing duration of the hospital stay.

\section{Pathology}

RSV is acquired during close contact with infected individuals or self-inoculation with infected secretions. Respiratory-tract infection develops via cell-to-cell transmission and syncytia formation. Incubation period is usually $2-$ $8 \mathrm{~d}$.

Initially there is necrosis of the respiratory epithelium and destruction of ciliated epithelial cells, followed by peribronchiolar infiltration with lymphocytes. There is variable degree of submucosal edema, but the collagen and elastic tissues are spared. Alveoli are usually spared but in severe cases increased cellularity of the intra-alveolar walls and some intra-alveolar edema fluid may be seen. Mucus plugs composed of the cellular debris and fibrin may occlude the smaller airway and cause distal patchy micro-atelactesis. During the recovery phase, bronchiolar epithelium regenerates more promptly and the ciliary growth follows a few days later.

Various immunologic and nonimmunologic factors play a role in RSV-induced bronchiolitis. Increased production of Th2-type cytokines with its inflammatory properties, interferon- $\gamma$ (IFN- $\gamma$ ) through induction of leukotrienes, and altered pulmonary surfactant have all been proposed in the pathogenesis (9).

\section{Pathophysiology}

Obstruction of the smaller airways owing to small mucus plugs and edema of the airway wall along with bronchospasm of the relatively narrow-diameter infant airways leads to significant increase in airway resistance. Patchy atelactesis and alveolar hyperinflation results. The functional residual capacity approximately doubles and breathing work is significantly increased as the infant breathes at high lung volumes with increased airway resistance and reduced dynamic lung compliance.

Arterial hypoxemia results mainly because of ventilation perfusion mismatching as the obstructed units continue to get perfusion but 


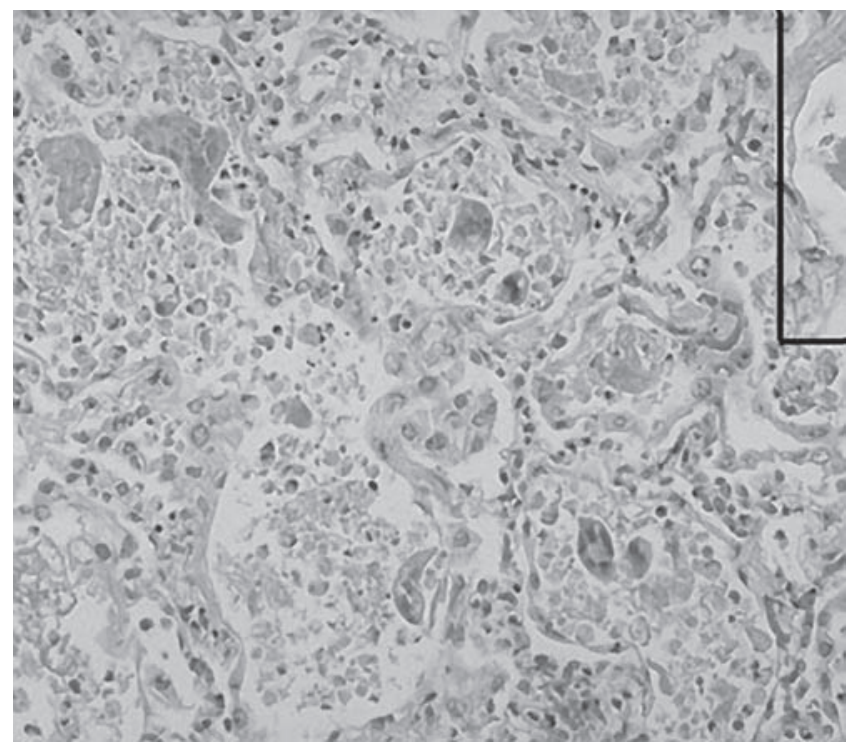

Fig. 1. RSV bronchiolitis. Note the giant cells, which are part of the viral cytopathic effect. The inset demonstrates a typical giant cell with a round, pink intracytoplasmic inclusion. (Web Path, University of Utah.)

hardly any ventilation, and the overdistended units continue to have the same or less blood supply owing to alveolar hypoxic vasoconstriction. This etiology is substantiated as supplemental oxygen corrects the hypoxemia. Increased work of breathing leads to respiratory exhaustion. Carbon dioxide retention is not seen until acute respiratory failure ensues. Initially some infants may show respiratory alkalosis owing to increased ventilation, but later, metabolic acidosis caused by increased respiratory muscle activity and poor fluid and caloric intake occurs. In cases with carbondioxide retention, a component of respiratory acidosis may be present. Apnea usually has a central component besides the obstruction owing to excessive upper-airway secretions.

\section{Clinical Manifestations}

Upper-respiratory symptoms with coryza, excessive sero-mucoid nasal secretions, and congestion usually occur $2-3 \mathrm{~d}$ before the abrupt onset of lower-respiratory distress. Cough, wheezing, increased rate and work of breath-
Table 2

Mechanisms of Airway Obstruction in Bronchiolitis

Small-airway diameter

Inflammation of the airway wall

Mucus plugging from the secretions and cellular debris

Smooth-muscle spasm

Altered neural control mechanisms Increased parasympathetic signal

Decreased nitric oxide (NO) production

ing with retractions occur as bronchiolitis sets in. Fever is not significant. Depending on the severity of the illness, continued respiratory distress leads to exhaustion and respiratory failure sets in. Poor feeding and cough associated vomiting can lead to dehydration and further complicate the clinical situation. Most infants have milder self-limiting disease and improvement begins approx 5-7 d into the illness. Severe presentations are seen in infants at high risk of respiratory disease, such as infants with chronic lung disease of prematurity, hemodynamically significant congenital heart disease, immunodeficiency, congenital malformation of the lungs, and cystic fibrosis (CF). Diagnosis relies on clinical recognition. Usually there is history of a sick contact or exposure in the day-care center.

Younger and smaller children between ages 1-4 mo more frequently require hospital admission. Severity is judged by respiratory distress, degree of hypoxia, and need for support ventilation. Exposure to environmental tobacco smoke and family history of asthma augment the risk for hospital admission.

Apnea with RSV infection is commonly seen in preterm infants who also have pulmonary hypertension related to chronic lung disease of prematurity. Apnea can precede, follow, or co-exist with bronchiolitis. It is rarely the sole manifestation of bronchiolitis. The exact mechanism of apnea is unclear and may have a central component. Recurrent apnea may require ventilatory support even in the absence 


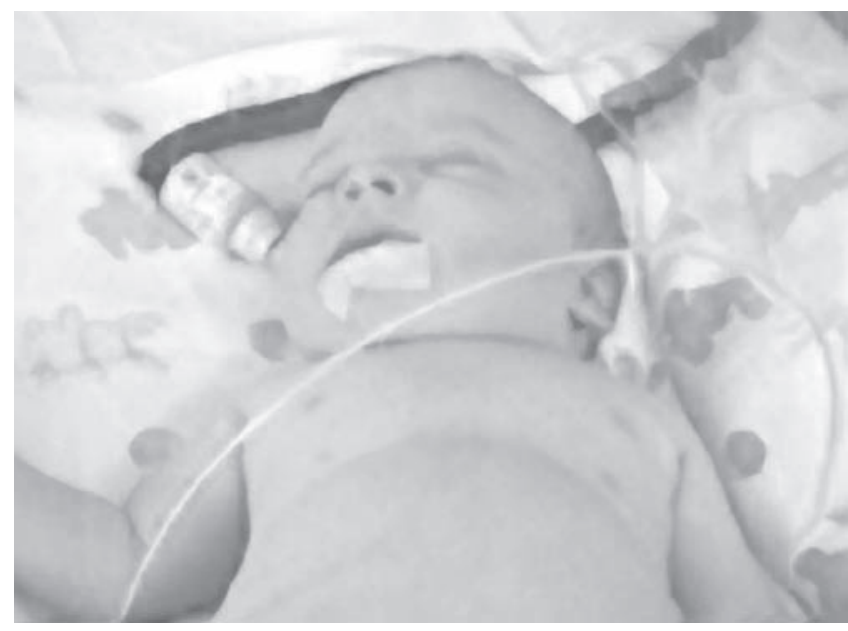

Fig. 2. Infant in respiratory distress with nasal flaring and chest retractions.

of respiratory failure. Fortunately apnea tends to resolve within few days.

Respiratory distress, chest retractions, cyanosis, agitation, audible wheeze, and low oxygen saturations usually precede the onset of respiratory failure as evidenced by increased rate and work of breathing. Physical exam of the infant or child chest wall may reveal intercostals retractions, increased anteroposterior diameter of the chest, and hyper-resonant chest percussion owing to hyper-inflated lungs. Inspiratory and expiratory rhonchi dominate the lung auscultation, although end inspiratory crackles may be heard in patients with simultaneous alveolar involvement. As the sickness continues, poor oral intake and excessive loss of moisture with rapid breathing leads to signs of dehydration. Poor weight gain and weight loss may occur. Rapid heart rate, feeding difficulty, excessive perspiration, and enlarging liver size may indicate onset of congestive heart failure (CHF).

Oxygen-saturation monitoring is of critical importance in most cases and the degree of oxygen desaturation correlates well with the severity of the disease. Hypercapnia is seen only in severe cases. Arterial blood gases are obtained only if intubation is being considered. Chest radiographs in severe bronchiolitis typi- cally show hyperinflated lung fields, patchy atelactesis, peri-hilar congestion, and frequently small areas of alveolar consolidation. There is no significant correlation between radiographic appearance and severity of bronchiolitis. Because coexisting bacterial lung infections are uncommon, patchy opacities on chest X-ray should not be used as an indication for antibiotic therapy. High white blood cell counts are infrequent, although some left shift attributable to stress factors might be seen.

Hospital admission is not needed for most infants except for the very young (less than 6 mo), those with risk factors (prematurity, congenital heart disease, etc.), and infants with poor clinical status (respiratory distress, dehydration, hypoxia, and apnea). Parents of the infants treated at home should be forewarned about the signs of deterioration such as poor feeding, increased distress, and lethargy.

\section{Diagnosis}

Besides a thorough history (especially contact sickness, day-care exposure, etc.), a complete physical exam (especially chest, lungs, and heart), a CBC with differential counts, basic electrolytes, and chest X-ray, detection of RSV and other viral antigens in nasal secretions is very helpful in making a precise etiologic diagnosis. Nasopharyngeal aspirates provide a high yield of virus antigen, as does the nasal lavage or nasal wash. A rapid diagnosis can be made using immunofluorescent antibody detection or enzyme-linked immunosorbent assay (ELISA). A positive result supports the diagnosis, although a negative result does not rule out the possibility with any certainty; therefore, careful attention should be paid to obtaining a good-quality sample.

RSV can be cultured in appropriate cell lines, but the results usually take up to a week and careful handling of the sample is needed. A nasal swab should be sent if a viral culture is being considered, making sure that enough cells are present. 
Table 3

Symptoms and Signs in Severe Bronchiolitis

Symptoms:

Excessive nasal secretions

Cough

Rapid breathing with increased effort

Poor feeding

Vomiting, diarrhea

Signs:

Tachycardia

Tachypnea

Low-grade fever

Nasal flaring and chest retractions

Cyanosis

Hyper-resonant chest percussion

Crackles and rhonchi with increased expiration

Signs of CHF such as diaphoresis and enlarging liver size

Apnea

Signs of dehydration such as poor urine output, lethargy

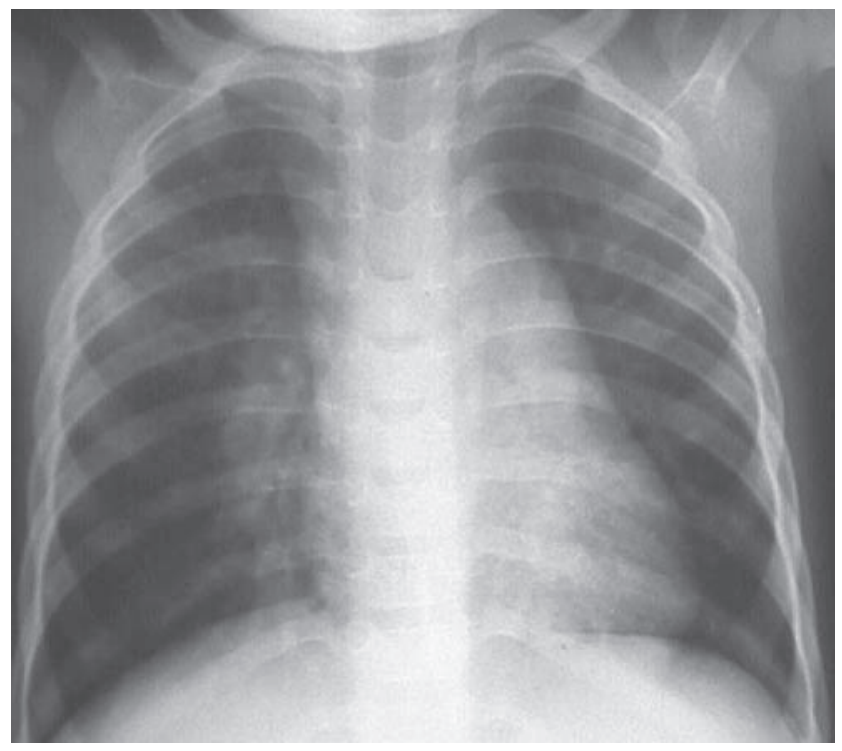

Fig. 3. Typical chest radiograph in bronchiolitis with hyper-inflated lung fields, hilar congestion, and patchy infiltrate.

\section{Differential Diagnosis}

The clinical picture of bronchiolitis is similar whether caused by RSV, parainfluenza, influenza, adenovirus, or rhinoviruses. A more severe atypical and prolonged form of the dis- ease is associated with other concomitant primary diagnoses such as $\mathrm{CF}$, congenital heart disease, immunodeficiency, congenital lung abnormality, recurrent aspiration syndromes, and bronchopulmonary dysplasia (BPD). It is important to differentiate bronchiolitis from pneumonia because about $20-25 \%$ patients with bronchiolitis may have areas of segmental atelactesis or consolidation on chest X-ray. In bronchiolitis, the lung fields are distinctly hyperinflated.

The initial clinical picture of severe bronchiolitis and acute severe asthma can look identical. A strong family history of asthma, eczema, or other atopic disorders, negative viral antigens in the nasal secretions, and most importantly repeated episodes of cough and wheezing without complete resolution make diagnosis of asthma more likely. Recurrent bronchiolitis mandate a further work-up for underlying pulmonary disease. History of chronic ETS exposure can make complete resolution of symptoms more difficult, and more severe bronchiolitis requiring hospital admissions is frequently observed in these instances.

\section{Management}

Vigilant monitoring and high-quality supportive care is the key to success. Most viral bronchiolitis is self-limited and therefore the primary management is aimed at symptomatic, supportive treatment with careful observation for apnea, hypoxia, and sheer exhaustion.

\section{Oxygen}

Hypoxia occurs mainly because of the ventilation-perfusion mismatching and can be treated by using 30-40\% oxygen via a head box, face mask, or nasal canula. A head box is more suitable for agitated, distressed infants.

\section{Fluid and Nutrition}

Impressive deficits in oral intake of fluids and nutrition can accumulate quickly in the respiratory distressed infant. Frank dehydra- 
tion, coexistent gastroesophageal reflux, and vomiting related to coughing can further complicate management. Adequate nutritional support and maintenance fluid via intravenous (iv) route should be provided. Because severe pulmonary involvement may be associated with inappropriate antidiuretic hormone secretion (SIADH), a close eye should be kept on serum sodium and the need to restrict the fluids to two-thirds of the maintenance. Conversely, fluid overload can cause pulmonary edema and increased rate and work of breathing. Oral and nasogastric feedings may be possible in less severe cases but are not recommended in the light of the respiratory distress, increased risk of gastroesophageal reflux, and aspiration.

\section{Bronchodilators}

Nebulized racemic albuterol or levalbuterol continues to be used in the acute management of severe bronchiolitis despite the absence of convincing evidence of their usefulness. Several studies have failed to show any benefit from the use of $\beta 2$ sympathomimetic bronchodilators with some possible detrimental effects. A review of 15 published studies concluded bronchodilators to be of modest benefit in mild to moderate bronchiolitis (10). Beneficial effects of these agents in some of the infants can be better defined if they are segregated into the wheezy and nonwheezy group, as the former is more likely to respond. Most of the younger (less than $6 \mathrm{mo}$ ) patients belong to the nonwheezy group and respond poorly to the bronchodilators. Thus for older infants a trial of the bronchodilator may be worthwhile because it may be difficult to distinguish bronchiolitis from the other diseases and disorders that feature wheezing. Infants with bronchopulmonary disease (BPD) and those with recurrent wheezing with a family history of asthma or other atopic disorders also respond better to bronchodilator therapy.

\section{Decongestants}

Short-acting nebulized racemic epinephrine has been shown to provide significant symptomatic relief and decreased the need for hospital admission in severely congested infants with bronchiolitis. It has been found to be superior to nebulized albuterol or placebo in relieving symptoms $(11,12)$. Epinephrine acts as a decongestant. Its alpha-adrenergic properties cause pulmonary vasoconstriction, reducing mucosal edema and exudates and thereby reducing airway obstruction. It is most helpful in the earlier part of the illness, but repeated use over a longer time may result in rebound congestion.

\section{Corticosteroids}

Glucocorticoids administered systemically or inhaled for their antiinflammatory property have failed to show convincing benefit clinically and do not decrease interleukin-8 (IL-8) secretion in infants with RSV bronchiolitis (13). In one study, a single dose of (14) dexamethasone was used in emergency room, and this resulted in fewer hospital admissions. Steroid use is more common if the diagnostic dilemma exists between bronchiolitis and early-onset asthma; it is also more common in intensive care and outpatient practice (15).

\section{Physiotherapy}

Mist therapy can further compromise the infant with frequent requirement for suctioning. Chest physiotherapy (CPT) requires frequent handling and has no proven benefit in clearing secretions from the smaller airways involved in bronchiolitis unless significant atelactesis is present.

\section{Antibiotics}

Antibiotics are not indicated unless the clinical picture is atypical, rapid RSV test is negative, and the severity of the disease is high, presenting as an apparent septicemic illness. Infants requiring intensive care unit admission 
and intubation are usually treated with penicillins or first-generation cephalosporins.

\section{Ventilatory Support}

Assisted ventilation with a variety of techniques may be required for recurrent apnea or the respiratory failure $(16,17)$. Indications for intubation and ventilation commonly include hypoxia and acidosis despite oxygen therapy, respiratory exhaustion, and recurrent apnea. Although airway resistance is significantly increased and high peak pressures are often required, minimum-pressure control settings that provide adequate oxygenation should be used. Permissive hypercapnia should be preferred over aggressive ventilatory strategy to normalize carbon dioxide. Carbon dioxide retention is not seen frequently except for the most exhausted infants. A generous expiratory time should be provided and rates should be kept low. Use of high positive end expiratory pressure (PEEP) may be detrimental in the older infants with wheezing. Small increase in PEEP may be supportive in younger infants without wheezing and in those with ventilatory exhaustion. Ventilatory support should be discontinued as early as possible unless there are other complications such as adult respiratory distress syndrome (ARDS). Infants who continue to deteriorate can benefit from extracorporeal membrane oxygenation (ECMO).

A few reports suggest that high-frequency ventilation combined with $\mathrm{NO}$ may be beneficial in those who do not respond to the conventional ventilation. Other reports suggest the usefulness of intermittent negative intrathoracic pressure in infants with apnea and the early use of continuous positive pressure (CPAP) to reduce need for more intubation and ventilatory support.

\section{Antileukotrienes}

Role of cysteinyl leukotrienes (cys-LT) has been implicated as one of the inflammatory mediators in bronchiolitis. Leukotrienes are upregulated in RSV bronchiolitis and also in patients exposed to ETS. Montelukast, a cys-LT receptor antagonist, has been shown reduce pulmonary symptoms from RSV bronchiolitis $(18,19)$. Research studies in emergency room and inpatient hospital settings are underway to ascertain if administration of montelukast can reduce the duration and severity of RSV bronchiolitis in infants.

\section{Antiviral Agents}

Ribavirin is a virustatic drug delivered as an aerosol. Poor efficacy, difficult administration, side effects, and toxicity limit its use. It requires a negative pressure room; can transiently irritate the airways, making cough worse; and has teratogenic potential to pregnant health-care providers exposed to the drug. It is no longer recommended except for individual cases with severe RSV-related disease where other options have failed.

\section{Prevention}

\section{Contact Precautions}

RSV is highly contagious and spreads rapidly upon contact with infected secretions; the droplets generated by coughing or sneezing do not play as important a role. Adequate hand washing by health-care providers reduces chances of self-inoculation and the direct transmission of virus to the other patients. Isolating an individual patient is not as effective as cohorting to limit the transmission. It is very important to consider all possible measures to prevent nosocomial spread, especially during an epidemic to protect the infants at highest risk. Contact transmission is also very significant in day-care centers and appropriate hygienic measures with frequent hand washing help prevent spread (20).

\section{Vaccines}

Active immunization using a live or killed vaccine, systemic or inhaled has not been rewarding so far. Earlier attempts during the 1960s using formalin-inactivated alum precipitated vaccine failed to protect and resulted in 
increased morbidity and mortality rate in the immunized infants. Experiments with more stable, attenuated, and immunogenic strains have shown to be effective in animal models, but efficacy in human infants still need to be proved. One major concern is the duration of protection, as even the natural infection does not confer a lasting or complete immunity, and re-infections are quite common.

Passive immunization using specific immunoglobulin via iv or the intramuscular (im) route provides a high level of the neutralizing antibodies and has proved to be protective (21-23). Protection is short-lived and the doses need to be repeated every month. Data suggest that using this type of protection in the high-risk infant can be rewarding particularly in reducing the need for hospital admission. These agents did not show any improvement in the hospital course when used as treatment. Two currently available preparations are Respigam (iv pooled anti-RSV IgG antibody) and Synagis (palivizumab, im monoclonal antibody [MAb] against RSV). The latter is preferred because of its low volume and lack of a need for of a hospital admission for the iv infusion. Although the popularity of this type of prevention is increasing, the cost is a limiting factor (24). The American Academy of Pediatrics (AAP) has published guidelines for its use in high-risk infants (25).

\section{Prognosis}

Prognosis for the majority of previously normal infants who develop bronchiolitis is good. The mortality rate is less than $1 \%$ in this group. In the high-risk group, such as those with congenital heart disease, mortality is less than $4 \%$.

Long-term effects of having had bronchiolitis as an infant include recurrent wheezing and asthma in as many as $75 \%$ patients. There is evidence that the prevalence declines with the age and is highest in the first 6 mo after the initial episode $(26,27)$. Presence of a personal or family history of asthma or other atopic disorders predisposes adults to recurrent wheezing, as does the exposure to the environmental tobacco smoke.

\section{Conclusion}

Although the mortality associated with viral bronchiolitis is much lower now with improved supportive care, morbidity still continues to be high. Viral bronchiolitis poses a major health threat to the high-risk infant. Use of antiRSV immunoglobulin and strict observation of respiratory-contact precautions along with the improved supportive care is the mainstay of treatment. Antiviral therapy with ribavirin has not proven to be as useful and has a very limited role at the present time.

\section{References}

1. Welliver, R.C. (2003), Pediatr Infect Dis J 22, S6-S12.

2. Simoes, E.A. (1999), Lancet 354, 847-852.

3. Staat, M.A. (2002), Semin Respir Infect 17, 15-20.

4. Van Woensel, J.B., Kimpen, J.L., and Brand, P.L. (2001), Minerva Pediatr 53, 99-106.

5. Krilov, L.R. (2001), Curr Infect Dis Rep 3, 242-246.

6. Hall, C.B. (1999), J Pediatr 135, 2-7.

7. Nicolai, T. and Pohl, A. (1990), Lung 168 Suppl, 396-405.

8. Pichler, M.N., Reichenbach, J., Schmidt, H., et al. (2000), Acta Paediatr 89, 1387-1389.

9. van Schaik, S.M., Welliver, R.C., and Kimpen, J.L. (2000), Pediatr Pulmonol 30, 131-138.

10. Kellner, J.D., Ohlsson, A., Gadomski, A.M., and Wang, E.E. (1996), Arch Pediatr Adolesc Med 150, 1166-1172.

11. Meates, M. (2002), Arch Dis Child 87, 548-550.

12. Bertrand, P., Aranibar, H., Castro, E., and Sanchez, I. (2001), Pediatr Pulmonol 31, 284-288.

13. Thomas, L.H., Sharland, M., and Friedland, J.S. (2002), Pediatr Res 52, 368-372.

14. Schuh, S., Coates, A.L., Binnie, R., et al. (2002), J Pediatr 140, 27-32.

15. Hvizdos, K.M. and Jarvis, B. (2000), Drugs 60, 1141-1178.

16. Gavin, R., Anderson, B., and Percival, T. (1996), N Z Med J-109, 137-139.

17. Lebel, M.H., Gauthier, M., Lacroix, J., et al. (1989), Arch Dis Child 64, 1431-1437.

18. Bisgaard, H. (2003), Am J Respir Crit Care Med 167, 379-383.

19. Ng, D.K., Law, A.K., Chau, K.W., and Chan, H.K. (2000), Respirology 5, 389-392.

20. Weisman, L.E. (2002), Pediatr Int 44, 475-480.

21. Romero, J.R. (2003), Pediatr Infect Dis J 22, S46-S54. 
22. Rodriguez, W.J., Gruber, W.C., Groothuis, J.R., et al. (1997), Pediatrics 100(6), 937-942.

23. Rodriguez, W.J., Gruber, W.C., Welliver, R.C., et al. (1997), Pediatrics 99, 454-461.

24. Barton, L.L., Grant, K.L., and Lemen, R.J. (2001), Pediatr Pulmonol 32, 20-28.
25. Committee on Infectious Diseases and Committee on Fetus and Newborn. (1998), Pediatrics 102(5), 1211-1216.

26. Schauer, U., Hoffjan, S., Bittscheidt, J., et al. (2002), Eur Respir J 20, 1277-1283.

27. Sigurs, N. (2002), Respir Res 3(Suppl 1), S8-S14. 